\title{
High Speed Braking Process Analysis of Monorail Rocket Sled Based on Fluid- Structure Interaction
}

\author{
Xuewen ZHOU ${ }^{\mathrm{a}}$, Shuiyan $\mathrm{LV}^{\mathrm{b}}$ and Xianwei LIU ${ }^{\mathrm{b}, 1}$ \\ ${ }^{a} X i^{\prime}$ an Jiaotong University, Xi'an 710049 China \\ ${ }^{\mathrm{b}}$ Rocket Sled Test Method Research Center, Test Institute of North Industries Group \\ Corporation, Xi'an 710018, China
}

\begin{abstract}
Water brake technology has great significance on monorail rocket sled test, which coupling nonlinear process between high-speed flow impact and structure response. In this paper Finite element method(FEM) and Smoothed particle hydrodynamics(SPH) model is used to analyze the braking force performance and the structure response during the monorail rocket sled braking process. The results show that the FEM-SPH method has high accuracy, which braking force error between numerical simulation and experiment is $3.92 \%$ when velocity of rocket sled is $750 \mathrm{~m} / \mathrm{s}$. The safe braking distance of numerical simulation is $100 \mathrm{~m}$, while the real distance of experiment is $105 \mathrm{~m}$. The error between numerical simulation and experiment is just about $5 \%$.
\end{abstract}

Keywords. Rocket sled, water brake, smoothed particle hydrodynamics

\section{Introduction}

Rocket sled test is a large-scale dynamic test system which takes the rocket engine as the power device to promote the test object to run on the high-precision orbit, so as to realize the simulation of high-altitude environment. It is of great significance for the verification of modern high-technology [1,2]. When the rocket sled reaches the end point with high speed, it needs to be braked by water bag. At this time, the huge impact between the sled moving with hypersonic speed and water may cause the strength failure of the component structure. Therefore, based on safety considerations, it is necessary to analyze the water braking process near the end point of the rocket sled.

Scholars at home and abroad have carried out a lot of researches on the numerical simulation method of fluid-structure coupling impact in the process of water braking. Wang et al. [3,4] used the fluid-structure coupling method to analyze the high-speed water impact process of rocket skid water brake. It is found that the supercavitation formed by water brake blade and side plate head has the greatest impact on water resistance, followed by water uplift, and basically has no impact on water splash. The stepped water entry structure of water brake can effectively reduce the peak value of water entry impact force. Gao et al. [5] based on star-ccm+ and ABAQUS platform, built the fluid-structure coupling numerical model of high-speed water inflow of rotating body,

${ }^{1}$ Corresponding Author, Xianwei LIU, Rocket Sled Test Method Research Center, Test Institute of North Industries Group Corporation, Xi’an 710018,China; Email: lxw_newslink@163.com 
and carried out the numerical calculation of high-speed inclined water inflow of rotating body with different water inflow velocities. Yu et al. [6] combined with the modal information provided by the finite element method and the analytical hydrodynamic model, built the hydroelastic analysis method of two-dimensional wedge water impact model. The calculation results under different grid density and modal number show that the method has good convergence. Yang et al. [7] used the moving particle semi-implicit method(MPS method) to simulate the fluid-structure interaction of flat bottom structure into water. The comparison between the simulation and experimental results shows that MPS method can be well applied to the numerical calculation of discontinuous problems such as impact into water. Stenius et al. [8] analyzed the fluid structure coupling mechanism of two-dimensional elastic wedge water impact by using LS-DYNA software.

Considering the dynamic response characteristics of rocket sled impacting water bag brake at high speed, in this paper, FEM is used to simulate the finite element model of rocket sled, and SPH particles are used to simulate the dispersion process of long cylindrical water particles during braking. The braking process with $1 / 3$ bag of water and full bag of water in $30 \mathrm{~mm}$ during braking is simulated and analyzed respectively, and the comparative analysis of experimental verification is carried out.

\section{Analysis Model in LSDYNA}

\subsection{Contact Algorithm Between Rocket Sled and Water Bag}

The fluid structure coupling algorithm in LS-DYNA program can be handled by three methods: First merging the interface nodes of fluid and structure. Second contact algorithm. Third Euler-Lagrange coupling algorithm. Merging the two-phase interface nodes makes the fluid and structure mesh have the same density and number of nodes at the two interfaces, so that the fluid and structure share the same interface and nodes. During the calculation, the fluid and structural mesh deform together. This method cannot be applied to this situation. Euler-Lagrange coupling algorithm has been applied in many large-scale finite element software, but the disadvantage of this algorithm is that the simulation process need a lot of computer resources and the numerical simulation time is too long. It also cannot be applied to this simulation. In this paper, the Lagrange two-phase particle method and finite element contact algorithm are used to analyze the braking process of monorail rocket sled, which should define the contact slip surface between the water bag and the rocket sled. The method is the same with the definition of collision contact type.

The sheet contact algorithm used in this paper is the symmetric penalty function method. Symmetric penalty function method is a relatively new algorithm, and its principle is rather simple. During each time step the method first checks whether each slave node penetrates the main surface, and if there is no penetration, the slave node will not be treated. In case of penetration, a large interface contact force is introduced between the slave node and the penetrated main surface. The force is directly proportional to the penetration depth and the stiffness of the main plate, which is called the penalty function value. Its physical meaning is equivalent to placing a normal spring between the main surface and the slave surface to limit the penetration of the slave node to the main surface. The symmetric penalty function method processes the master node again at the same time, and the filter algorithm is the same as that of the slave node. The symmetric penalty function method is simple to program, rarely arouses the hourglass effect of the grid and 
has no noise. This is because the algorithm has symmetry, accurate momentum conservation, no collision and release conditions, and the size of the penalty function is limited by stability. If obvious penetration is found in the calculation, it can be adjusted by enlarging the penalty function value or reducing the time step.

\subsection{Finite Element Model}

The rocket sled grid includes hexahedron and prism cell. The model does not contain any tetrahedron and pyramid meshes. SPH particles are divided by LSDYNA's Prepost. The number of nodes varies with the thickness of the water bag. The particles are evenly divided at an interval of $5 \mathrm{~mm}$. The model is shown in the figure 1.

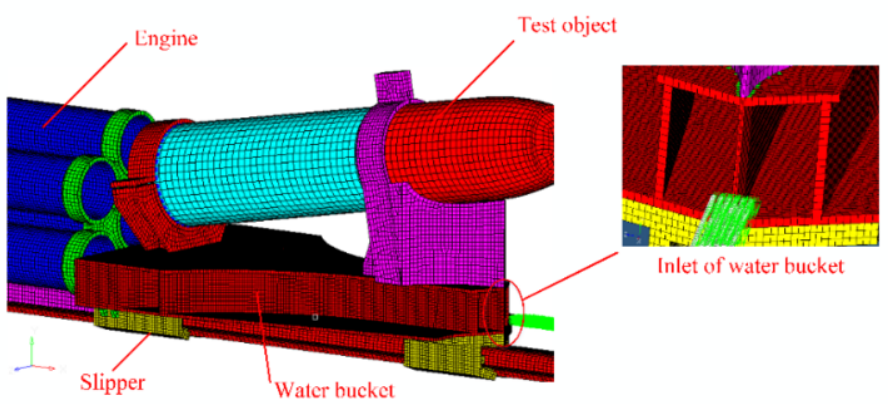

Figure 1. Whole grid of monorail rocket sled

As shown in figure 1, the monorail rocket sled mainly contains test object, engine, water bucket and slipper. The water bag is put on the rail while the water bucket pass fast, a great braking force can be imposed on the sled. The material properties of water bucket are shown in table 1.

Table 1. The material properties of water bucket

\begin{tabular}{cc}
\hline Material properties & Value \\
\hline Density & $7850 \mathrm{~kg} / \mathrm{m}^{3}$ \\
Elastic modulus & $2.0 \times 10^{11} \mathrm{~Pa}$ \\
Poisson's ratio & 0.284 \\
\hline
\end{tabular}

The properties of water can be described by the state equation below,

$$
p=\frac{\rho_{0} C^{2} \mu\left[1+\left(1-\frac{\gamma_{0}}{2}\right) \mu-\frac{a}{2} \mu^{2}\right]}{\left[1-\left(S_{1}-1\right) \mu-S_{2} \frac{\mu^{2}}{\mu+1}-S_{3} \frac{\mu^{3}}{(\mu+1)^{2}}\right]^{2}}+\left(\gamma_{0}+\mu\right) E
$$

The parameter values in the equation (1) are shown in table 2.

Table 2. The water properties

\begin{tabular}{cc}
\hline Properties & Value \\
\hline Density $\rho_{0}$ & $998 \mathrm{~kg} / \mathrm{m} 3$ \\
Truncature pressure E & $-2.2 \times 1010 \mathrm{~Pa}$ \\
Kinematic viscosity $\mu$ & $8.97 \times 10-7 \mathrm{~m} 2 / \mathrm{s}$ \\
$\mathrm{C}$ & 1480 \\
$\mathrm{~S} 1$ & 2.56 \\
$\mathrm{~S} 2$ & -1.99 \\
$\mathrm{~S} 3$ & 0.2268 \\
$\gamma$ & 0.5 \\
\hline
\end{tabular}




\section{Numerical Simulation and Experiment Results Analysis}

\subsection{Simulation Motion Process and the Fluid-Structure Interaction Analysis}

According to the aerodynamic simulation results, The aerodynamic force on the rocket sled is $59568 \mathrm{~N}$, the brake water bag diameter is $30 \mathrm{~mm}$, and the velocity of the rocket sled is $750 \mathrm{~m} / \mathrm{s}$. According to test requirements, the $30 \mathrm{~mm}$ water bag only contains $1 / 3$ bag of water, and the initial braking is carried out when the length of the water bag is $1.5 \mathrm{~m}$. The braking process is shown in the figure 2. Based on the space limit, the movement process of full bag of water braking is sketched.

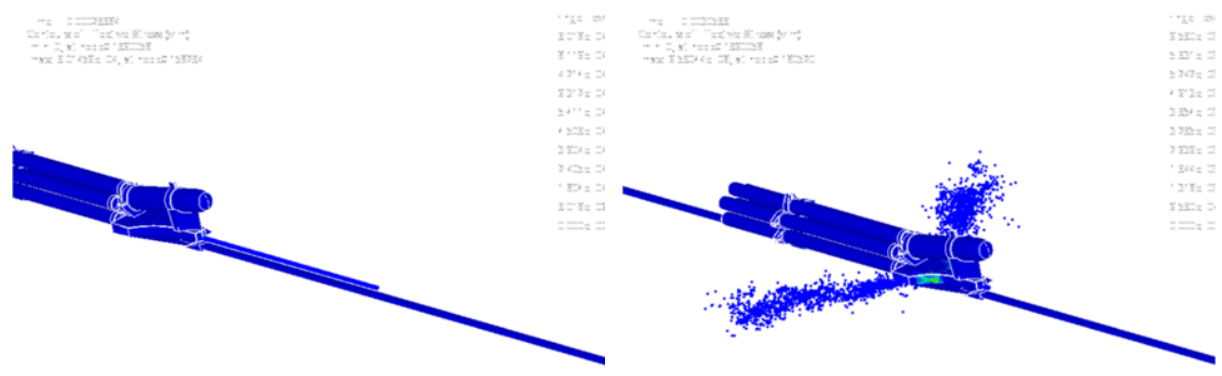

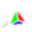

(a) $\mathrm{t}=0.3 \mathrm{~ms}$

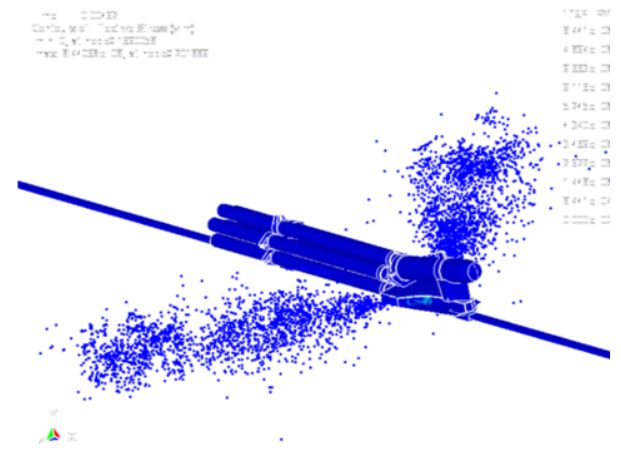

(c) $4.6 \mathrm{~ms}$ (b) $3 \mathrm{~ms}$

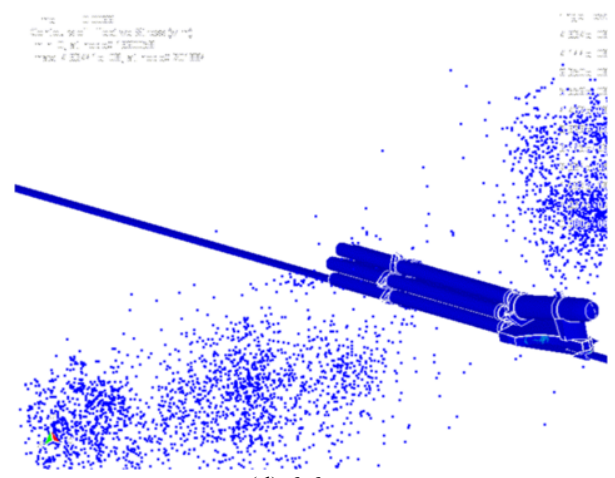

(d) $6.6 \mathrm{~ms}$

Figure 2. The water braking process of length $1.5 \mathrm{~m}$ diameter $30 \mathrm{~mm}$

As shown in the figure 2, the skid body enters the water braking stage at $0.3 \mathrm{~ms}$, and the stress of the middle plate of the bucket for dividing the water bag rises sharply to $510 \mathrm{mpa}$. With the advancement of the whole skid, the water flow is divided into two ways by the bucket and flows out of the bucket. The stress and strain on the deflector is large. The strain cloud diagram is shown in figure 3. The two places with the largest deformation are located in the middle plate and flow deflector of the dividing water bag respectively. In the actual test, water bags with different water volume are arranged according to different positions and speeds of the rocket skid. At the position of full water bag, the rocket skid is the most dangerous working condition. In this paper, only the stress-strain analysis of the most dangerous working condition is carried out. The fluidstructure analysis results of $30 \mathrm{~mm}$ diameter water bag are shown in figure 3 . 


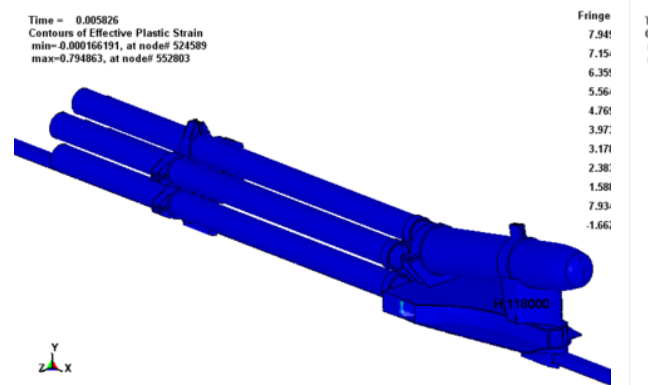

(a) The deformation of the sled
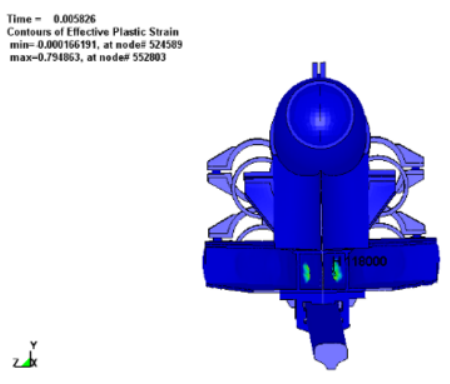

(b) Local deformation

Figure 3. Strains Distribution of the rocket sled

As shown in figure 3, the maximum shape variable of the water bucket is $0.8 \mathrm{~mm}$, which is located at the initial turning of the water bucket deflector. Strength check results of fluid-solid coupling simulation of $30 \mathrm{~mm}$ diameter water bag are shown in figure 4 .

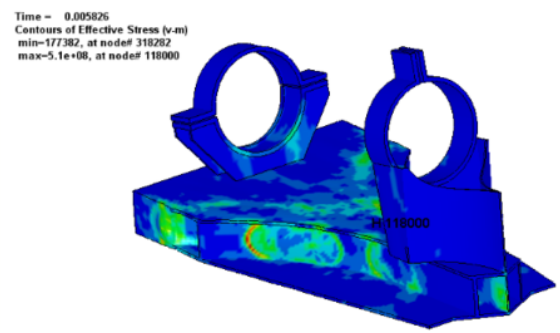

$\operatorname{Lu}_{\mathrm{x}}$

(a) Synthetic stress of water bucket at the moment of entering water brake

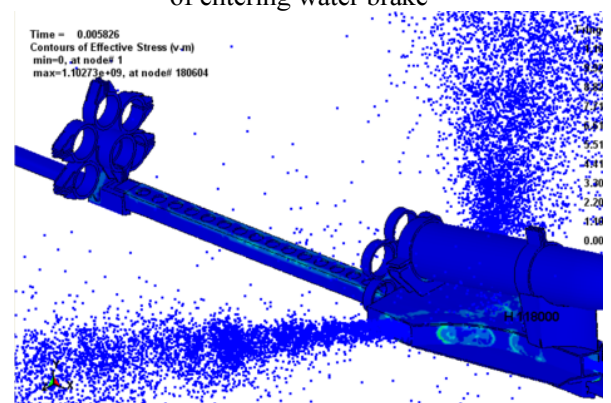

(c) Combined stress of rocket sled system

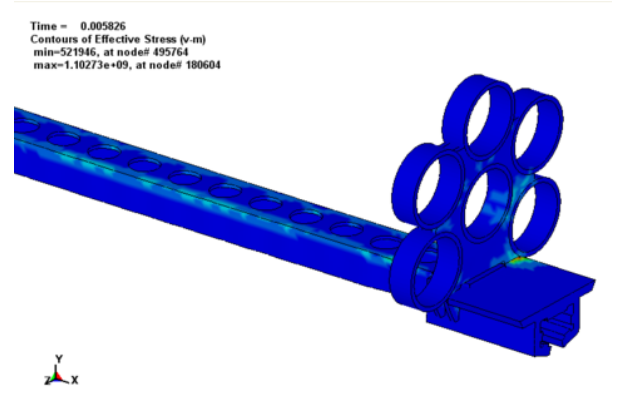

(b) Local composite stress of sled system

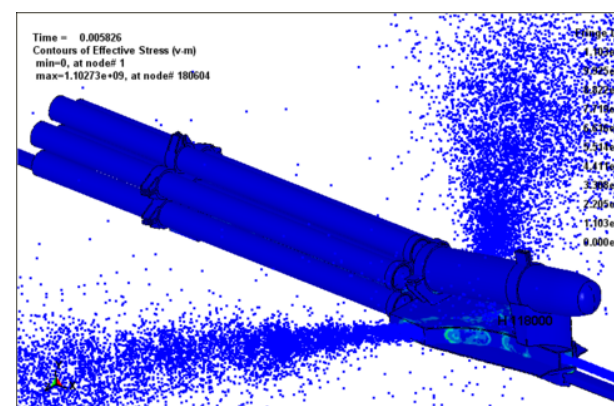

(d) Combined stress of rocket sled system

Figure 4. Stress Distribution of the rocket sled

It can be seen from the figure 4 that the maximum stress occurs at the connection between the front snap ring of the propulsive part and the slipper, with the maximum stress of $1300 \mathrm{MPa}$. The water bucket has a large stress on the water impact turning deflector, with the maximum stress of $1100 \mathrm{MPa}$. The overall synthetic stress of other parts of the skid in this state is below $900 \mathrm{MPa}$, which is lower than the yield strength of the sled material. 


\subsection{Experiment Results Analysis}

The sled is a monorail sled with the weight of $480 \mathrm{Kg}$. The brake segment is composed of water bags and plastic foam supports. The way to lay the water bag is to put the plastic foam bracket on the track first and put the water bag on the plastic support. The water bag is arranged as a monorail $950 \mathrm{~m}$, and the test trajectory diagram is shown in figure 5 .

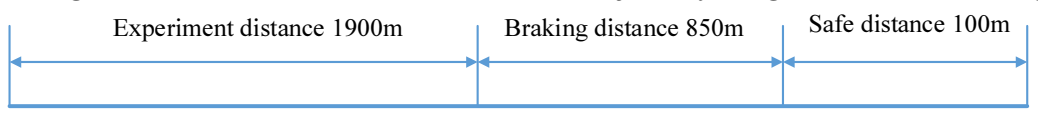

Strarting point

End point

Figure 5. Schematic diagram of test trajectory

According to the test of the overload of rocket sled, the sledge body gets into the special position of the braking section at the time of the skid resistance $F$, minus the aerodynamic drag simulation value, the friction force of the sliding shoes and the empirical value of the foam plate resistance, and obtains the $\mathrm{F}$ water brake force $\mathrm{F}_{\text {brake }}$. The brake force results during braking process are shown in the figure 6 .

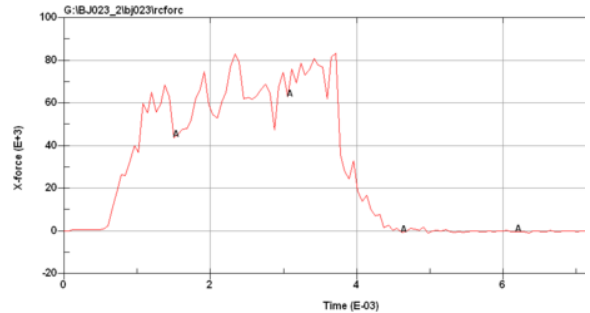

(a) Braking force of $1 / 3$ water bag

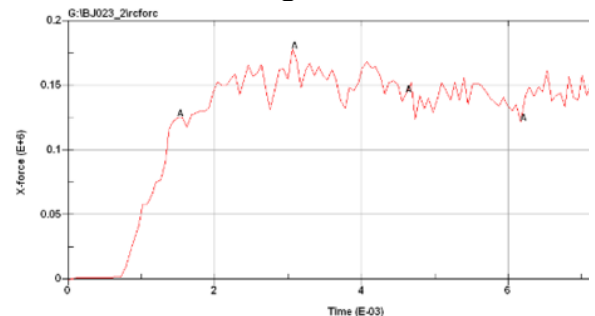

(b) Braking force of full water bag

Figure 6. Simulation results of braking force

During the simulation process, the speed of the rocket sled under the action of water brake resistance gradually decreases, and the water brake resistance has pulsating response. Therefore, the braking force is taken as the average value of the force within $1 \mathrm{~ms}$ after the water completely enters the bucket. Comparison between numerical and experimental results of braking force is shown in table 3 .

Table 3. The braking force between numerical and experimental results

\begin{tabular}{ccccc}
\hline Water volume & Speed(m/s) & $\begin{array}{c}\text { Experimental } \\
\text { braking force(N) }\end{array}$ & $\begin{array}{c}\text { Simulation } \\
\text { braking force(N) }\end{array}$ & Relative error \\
\hline $1 / 3$ water bag & 750 & $7.75 \times 104$ & $7.45 \times 104$ & $3.92 \%$ \\
Full water bag & 590 & $18.30 \times 104$ & $17.2 \times 104$ & $6.01 \%$ \\
\hline
\end{tabular}

During the experiment process, water bags with different diameters are arranged according to different positions and corresponding speeds of the rocket sled. According to different working conditions, the braking distance is calculated from the simulation results to be $850 \mathrm{~m}$. Considering the safety, $100 \mathrm{~m}$ allowance is reserved. In the real experiment, $105 \mathrm{~m}$ allowance is left for the rocket sled braking, and the simulation value is in good agreement with the test value. 


\section{Conclusion}

In this paper, the finite element smooth particle hydrodynamics method is used to simulate the braking process, braking force characteristics and water bag impact response of a monorail skid. The conclusions are as follows:

(1) The sheet contact algorithm is adopted in the research, which greatly reduces the search scope of the contact algorithm, saves the cost of computer memory and greatly reduces the computer time;

(2) The numerical simulations and test results of the rocket sled impacting the water bag at the speeds of $750 \mathrm{~m} / \mathrm{s}$ and $590 \mathrm{~m} / \mathrm{s}$ show that the method used in the research has high accuracy, and the braking force errors are only $3.92 \%$ and $6.01 \%$;

(3) The structural stress analysis of the sled shows that the water bucket has a large stress on the flow impact turning deflector, the maximum stress is $1100 \mathrm{MPa}$, the maximum stress occurs at the connection between the front snap ring of the thrust sled and the sliding shoe, and the maximum stress is $1300 \mathrm{MPa}$;

(4) The numerical analysis results show that more than $100 \mathrm{~m}$ safety distance should be reserved during the rocket sled test, which is consistent with the $105 \mathrm{~m}$ safety distance allowance in the actual test.

\section{References}

[1] Zhang J H and Jiang S S 2012 Rigid-flexible coupling model and dynamic analysis of rocket sled Advanced Material Research vol 2012 pp 447-454.

[2] Wang Y 2010 Development and prospect of rocket sled test Aviation Science And Technology vol $1 \mathrm{pp}$ $30-33$.

[3] Wang J, Zhao Q B, Tao G and Wu J J 2010 Numerical simulation of high speed water entry impact of rocket skid water brake Explosion And Shock Waves vol 30 pp 628-632.

[4] Wang J, Zhang X G and Kong W H 2013 Safety analysis on rocket sled water brake high-speed waterentry impact Journal Of Nanjing University Of Science And Technology vol 37 pp 902-906.

[5] Gao Y J, Sun T Z, Zhang G Y, You T Q Yin Z H and Zong Z 2020 Flow characteristics and structure response of high-speed oblique water-entry for a revolution body Explosion And Shock Waves vol 40 pp 123301 1-13.

[6] Yu P G, Wang T L, Zhao Y, Su S J Zhen C B 2019 Water entry of 2-D wedge by hydroelastic theory Journal Of Harbin Engineering University vol 40 pp 689-695.

[7] Yang C and Zhang H X 2018 Simulation of fluid-solid coupling problem during water-entry impact using moving-particle semi-implicit method Journal Of Zhejiang University(Engineering Science) vol $52 \mathrm{pp}$ 2092-2097.

[8] Stenius I, Rosen A and Kuttenkeuler J 2011 Hydroelastic interaction in panel-water impacts of high speed craft Ocean Engineering vol 38 pp 371-381. 